\title{
Mass-gap in the compactified principal chiral model
}

\author{
Jarah Evslin \\ Institute of Modern Physics, NanChangLu 509, Lanzhou 730000, China \\ and University of the Chinese Academy of Sciences, YuQuanLu 19A, Beijing 100049, China \\ Baiyang Zhang \\ Wigner Research Centre, H-1525 Budapest 114, P.O.B. 49, Hungary
}

(Received 25 July 2018; published 18 October 2018)

\begin{abstract}
If the space of minima of the effective potential of a weakly coupled $2 \mathrm{~d}$ quantum field theory is not connected, then a mass-gap will be nonperturbatively generated. As examples, we consider two $\sigma$ models compactified on a small circle with twisted boundary conditions. In the compactified $\mathbb{C P}^{1}$ model, the vacuum manifold consists of two points and the mass-gap is nonperturbative. In the case of the compactified SU(2) principal chiral model, the vacuum manifold is a single circle and the mass-gap is perturbative.
\end{abstract}

DOI: 10.1103/PhysRevD.98.085016

The similarity between the topological structure of fractional instantons in the two-dimensional $\mathbb{C P}^{1}$ sigma model and in Yang-Mills theory has long led to speculations that they play (distinct) roles in the generation of the mass-gaps of both theories [1]. Intriguingly, a similar half-charged excitation appears to cause the mass-gap in the $\mathrm{SU}(2)$ principal chiral model (PCM), where the Euclidean theory has no topologically stable solutions. More precisely, the mass-gap has been found analytically [2] and on the lattice [3] to be proportional to the strongcoupling scale which is the exponential of half of the action of the uniton saddle point found in Ref. [4]. Recently, in two remarkable papers $[5,6]$, the authors have proposed a new window on this puzzle. They claim that a weakly coupled circle compactification of the PCM with certain boundary conditions is adiabatically connected to the original model (see also Ref. [7]).

Needless to say, if crossed, the adiabatic bridge constructed by the authors may allow the mass-gap of the PCM to be understood and perhaps to shed light on confinement in Yang-Mills. As a first step in this direction, in the current paper, we will attempt to understand the weakly coupled (small circle) side of this bridge. We find several surprises with respect to its expected properties. We apply the same analysis to the $\mathbb{C P}^{1}$ model, whose adiabatic compactification was introduced in Refs. [8,9]. The Hamiltonian that we find for the resulting quantum mechanics is similar to but

Published by the American Physical Society under the terms of the Creative Commons Attribution 4.0 International license. Further distribution of this work must maintain attribution to the author(s) and the published article's title, journal citation, and DOI. Funded by SCOAP ${ }^{3}$. distinct from that found in Ref. [8]. This Hamiltonian provides a starting point for future investigations of a nonperturbative nature of the adiabatically compactified $\mathbb{C P}^{1}$ model.

The SU(2) principal chiral model is a $\sigma$ model whose target space is the group manifold $\mathrm{SU}(2)$. Let $U$ be the $\mathrm{SU}(2)$-valued field. Consider the $\sigma$ model compactified on a circle of circumference $L$ with the adiabatic twisted boundary conditions of Refs. [5,6]:

$$
U\left(\frac{L}{2}\right)=\sigma_{3} U\left(-\frac{L}{2}\right) \sigma_{3}
$$

where $\sigma_{3}$ is the third Pauli matrix and the time dependence is implicit. This boundary condition is easily visualized using the Hopf coordinates

$$
\begin{aligned}
& U=\left(\begin{array}{cc}
z_{1} & i z_{2} \\
i \overline{z_{2}} & \overline{z_{1}}
\end{array}\right), \quad z_{1}=\cos (\theta) e^{i \phi_{1}} \\
& z_{2}=\sin (\theta) e^{i \phi_{2}}, \quad \theta \in[0, \pi / 2], \quad \phi_{i} \in[0,2 \pi],
\end{aligned}
$$

where it is just

$$
\phi_{2}\left(\frac{L}{2}\right)=\phi_{2}\left(-\frac{L}{2}\right)+\pi
$$

The boundary condition is trivial when $U$ commutes with $\sigma_{3}$, corresponding to the circle

$$
U=\exp \left(i \phi_{1} \sigma_{3}\right)
$$


or equivalently to the circle $\left(\theta, \phi_{1}\right)=\left(0, \phi_{1}\right)$, where the $\phi_{2}$ circle degenerates.

As described in Ref. [6], the twisted boundary conditions increase the energy of a configuration away from these fixed points, and so lead to a potential for $\theta$. Classically, this potential vanishes precisely at the fixed point set of the symmetry $\phi_{2} \rightarrow \phi_{2}+\pi$, and so the circle (3) is the classical vacuum manifold of this theory. It is connected.

What about the minima of the effective potential, obtained by integrating out the oscillations transverse to this vacuum manifold? In principle, $\phi_{1}$-dependent masses for these transverse oscillations could lead to a $\phi_{1}$-dependent effective potential. This new potential could lift part of the circle, leaving a space of minima which is no longer connected. However, in the case at hand, both the action and the boundary condition are invariant under shifts $\phi_{1} \rightarrow \phi_{1}+c$. As we are in two dimensions, spontaneous symmetry breaking is forbidden [10] and so this symmetry is also respected by the effective action. Therefore, the minima of the effective potential will have the same symmetry. In the weakly coupled limit, the minima of the effective potential must be a nontrivial subset of the classical vacuum manifold, but the only such subset preserving the shift symmetry is the entire circle. Therefore, the space of minima of the effective action is a circle, which is connected, and so it does not satisfy the criterion describing the abstract for a nonperturbative mass-gap.

This is not to exclude nonperturbative contributions to the mass-gap. Indeed, such contributions are expected. However, as the space of minima is compact, we expect perturbative contributions to the mass-gap. As this theory is weakly coupled, the perturbative contributions will be far larger than the nonperturbative contributions, and so we say that the mass-gap is perturbatively generated. Below we will calculate these perturbative contributions explicitly and see that they are nonvanishing.

In Ref. [6], the authors use the Hopf coordinates with the fundamental domain $\theta \in[0, \pi], \phi_{1} \in[0, \pi], \phi_{2} \in[0,2 \pi]$. In these coordinates, the boundary condition is still given by Eq. (2). However, now the fixed point set is $\sin (\theta)=0$, where $\phi_{2}$ degenerates. In terms of $\theta$ and $\phi_{1}$, this consists of two intervals $\left(\theta=0, \phi_{1} \in[0, \pi]\right)$ and $\left(\theta=\pi, \phi_{1} \in[0, \pi]\right)$. It was claimed that there are two near degenerate vacua which are supported on these two intervals with even and odd parity under the symmetry $\theta \mapsto \pi-\theta$. However, the points $\left(\theta, \phi_{1}\right)=(0, \pi)$ and $\left(\theta, \phi_{1}\right)=(\pi, 0)$ both correspond to the same point $\left(z_{1}, z_{2}\right)=(-1,0)$, while both $(\theta, \phi)=(0,0)$ and $\left(\theta, \phi_{1}\right)=(\pi, \pi)$ correspond to the same point $\left(z_{1}, z_{2}\right)=(1,0)$; therefore, these two intervals are connected at their end points. The union of these two intervals is a circle; indeed it is just the vacuum manifold found using the fundamental domain in Eq. (2). The excitations of fields on this circle correspond to the states of a particle in a periodic box. In particular, a state which is odd under $\theta \mapsto \pi-\theta$, or equivalently $\phi_{1} \mapsto \phi_{1}+\pi$, will correspond to an odd excitation of the particle in a box, while the ground state is an even function. This splitting is perturbative, and in fact requires no deep excursions into the classically forbidden zone in which $\sin (\theta)>0$.

As was shown in Ref. [6], at small $L$ this theory is weakly coupled and the probability for the particle to venture far from the fixed point is exponentially suppressed. The interactions correspond to the curvature of the geometry and so the weak-coupling limit corresponds to a flattened neighborhood of the fixed circle. More precisely, in the small $L$ limit the target space becomes $\mathbb{C} \times S^{1}$, where $z_{2}$ is a coordinate of the $\mathbb{C}$ and $\phi_{1}$ is a coordinate of the $S^{1}$. The $\mathbb{C}$ and $S^{1}$ sectors are decoupled from each other at weak coupling. The twisted boundary conditions only affect the $\mathbb{C}$, where they yield $z_{2}(L / 2)=-z_{2}(-L / 2)$.

Expanding $z_{2}=y_{1}+i y_{2}$, the boundary condition becomes $y_{i}(L / 2)=-y_{i}(-L / 2)$. From the action

$$
\begin{aligned}
S & =\frac{1}{2 g^{2}} \int d x d t \operatorname{Tr}\left(\partial_{\mu} U^{\dagger} \partial^{\mu} U\right) \\
& =\frac{1}{g^{2}} \int d x d t\left(\partial_{\mu} \phi_{1} \partial^{\mu} \phi_{1}+\sum_{i} \partial_{\mu} y_{i} \partial^{\mu} y_{i}\right),
\end{aligned}
$$

one can find the canonical momenta

$$
\pi=\frac{2}{g^{2}} \partial_{t} \phi_{1}, \quad \Pi_{i}=\frac{2}{g^{2}} \partial_{t} y_{i} .
$$

The quantization of $\phi_{1}$ is just that of a particle in a periodic box. Suppressing time dependence, $\phi_{1}$ can be Fourier expanded on the compactified circle $x$ :

$$
\begin{aligned}
\phi_{1} & =\phi_{1}^{(0)}+\sum_{n \neq 0} \frac{1}{\sqrt{2 \frac{2 \pi}{L} n}}\left(a_{n}+a_{-n}^{\dagger}\right) e^{i \frac{2 \pi x}{L} n} \\
\pi & =\pi^{(0)}-\frac{2 i}{g^{2}} \sum_{n \neq 0} \sqrt{\frac{2 \pi n}{2 L}}\left(a_{n}-a_{-n}^{\dagger}\right) e^{i \frac{2 \pi x}{L} n} .
\end{aligned}
$$

Imposing $\left[\phi_{1}\left(x_{1}\right), \pi\left(x_{2}\right)\right]=i \delta\left(x_{1}-x_{2}\right)$ yields the commutation relations

$$
\left[\phi_{1}^{(0)}, \pi^{(0)}\right]=\frac{i}{L}, \quad\left[a_{m}, a_{n}^{\dagger}\right]=\frac{g^{2}}{2 L} \delta_{m n} .
$$

Normal ordering the Legendre transform, one obtains the Hamiltonian

$$
H=\frac{g^{2} L}{4} \pi^{(0)} \pi^{(0)}+\frac{4 \pi}{g^{2}} \sum_{n \neq 0}|n| a_{n}^{\dagger} a_{n} .
$$

Let the vacuum state be annihilated by both $a_{n}$ and $\pi^{(0)}$. Then there will be two families of rising operators which create excited states. First, $e^{i n \phi_{1}^{(0)}}$ is well defined for $n$ an 
integer as $\phi_{1}$ is $2 \pi$-periodic. These are the excited oscillator states of Ref. [6] and, in agreement with Eq. (5.18), their energy is

$$
\left[H, e^{i n \phi_{1}^{(0)}}\right]=E_{n} e^{i n \phi_{1}^{(0)}}, \quad E_{n}=\frac{g^{2} n^{2}}{4 L},
$$

which is the perturbative result that one expects for a particle in a box. Note that the lowest level state which is odd under $\phi_{1} \mapsto \phi_{1}+\pi$ is the state $n=1$, yielding a massgap of $g^{2} / 4 L$. This is our main result: the mass-gap is perturbative.

The Kaluza-Klein (KK) modes also yield excited states, created by $a_{n}^{\dagger}$. Their energy is given by

$$
\left[H, a_{n}^{\dagger}\right]=E_{n}^{\prime} a_{n}^{\dagger}, \quad E_{n}^{\prime}=2 \pi \frac{n}{L} .
$$

Note that $E^{\prime}$ is $g$ independent, unlike $E$, and so in the small $g$ or equivalently the small $L$ limit, these KK modes are much heavier than the particle in a box excitations.

The antiperiodic boundary conditions on the fields $y_{i}$ yield the Fourier decomposition

$$
\begin{aligned}
y_{i} & =\sum_{n} \frac{1}{\sqrt{2 \frac{2 \pi}{L}\left(n+\frac{1}{2}\right)}}\left(b_{i, n+\frac{1}{2}}+b_{i,-n-\frac{1}{2}}^{\dagger}\right) e^{i \frac{2 \pi x}{L}\left(n+\frac{1}{2}\right)} \\
\Pi_{i} & =-\frac{2 i}{g^{2}} \sum_{n} \sqrt{\frac{2 \pi}{2 L}\left(n+\frac{1}{2}\right)}\left(b_{i, n+\frac{1}{2}}-b_{i,-n-\frac{1}{2}}^{\dagger}\right) e^{i \frac{2 \pi x}{L}\left(n+\frac{1}{2}\right)} .
\end{aligned}
$$

Again the commutation relations of the quantum mechanical modes follow from those of the quantum fields:

$$
\begin{aligned}
{\left[y_{i}\left(x_{1}\right), \Pi_{j}\left(x_{2}\right)\right] } & =i \delta_{i j} \delta\left(x_{1}-x_{2}\right) \\
{\left[b_{i, m+\frac{1}{2}}, b_{j, n+\frac{1}{2}}^{\dagger}\right] } & =\delta_{i j} \delta_{m n} \frac{g^{2}}{2 L} .
\end{aligned}
$$

One then finds the Hamiltonian as above:

$$
\begin{aligned}
H & =\int d x \sum_{i}\left(\frac{g^{2}}{4}: \Pi_{i} \Pi_{i}:+\frac{1}{g^{2}}: \partial_{x} y_{i} \partial_{x} y_{i}:\right) \\
& =\frac{4 \pi}{g^{2}} \sum_{i, n}\left|n+\frac{1}{2}\right| b_{i, n+\frac{1}{2}}^{\dagger} b_{i, n+\frac{1}{2}} .
\end{aligned}
$$

Excited states are created with $b_{i, n+\frac{1}{2}}^{\dagger}$, each of which increases the energy by $\bar{E}_{n}$ :

$$
\left[H, b_{i, n+\frac{1}{2}}^{\dagger}\right]=E_{n} b_{i, n+\frac{1}{2}}^{\dagger}, \quad E_{n}=\frac{4 \pi}{L}\left(n+\frac{1}{2}\right) .
$$

Now we turn to the $\mathbb{C P} \mathbb{P}^{1}$ model. Note that the $y_{i}$ alone also describe the weak-coupling limit of the $\mathbb{C P}^{1}$ sigma model with antiperiodic boundary conditions introduced in Refs. [8,9]. As $\mathbb{C P} \mathbb{P}^{1}$ is $S^{2}$ and $\mathrm{SU}(2)$ is an $S^{3}$, one can pass from one model to the other via the Hopf projection $S^{3} \rightarrow S^{2}$, which identifies $\left(\phi_{1}, \phi_{2}\right) \sim\left(\phi_{1}+\alpha, \phi_{2}+\alpha\right)$. The invariant angle $\phi=\phi_{1}-\phi_{2}$ is the azimuthal coordinate of the $S^{2}$ and as such it degenerates at the poles $\theta=0$ and $\theta=\pi / 2$. The twisted boundary conditions are $\phi(L / 2)=\phi(-L / 2)+\pi$ and so are trivial at the two poles, which are the classical vacua of the theory. At weak coupling or more precisely small $L$, each of these classical vacua is described by the $y_{i}$ theory described above.

We can describe these two weak-coupling vacua explicitly by decomposing the field $y_{i}$ into KK modes, the degrees of freedom in the corresponding quantum mechanics,

$$
\begin{aligned}
y_{i} & =\sum_{n} y_{i, n+\frac{1}{2}} e^{i \frac{2 \pi x}{L}\left(n+\frac{1}{2}\right)} \\
\Pi_{i} & =\sum_{n} \Pi_{i, n+\frac{1}{2}} e^{i \frac{2 \pi x}{L}\left(n+\frac{1}{2}\right)},
\end{aligned}
$$

whose commutation relations yield a simple Schrödinger representation:

$$
\begin{aligned}
{\left[y_{i, m+\frac{1}{2}}, \Pi_{j, n+\frac{1}{2}}\right] } & =\delta_{i j} \delta_{m,-n} \frac{i}{L} \\
\Pi_{i, n+\frac{1}{2}} & =-\frac{i}{L} \frac{\partial}{\partial y_{-n-\frac{1}{2}}} .
\end{aligned}
$$

The vacuum must be annihilated by all of the $b$ 's,

$$
\begin{aligned}
0 & =b_{i, n+\frac{1}{2}}|0\rangle \\
& \propto\left[2 \pi g^{2}\left(n+\frac{1}{2}\right) y_{i, n+\frac{1}{2}}+\frac{\partial}{\partial y_{i,-n-\frac{1}{2}}}\right]|0\rangle,
\end{aligned}
$$

and so it is proportional to

$$
\psi=\exp \left[-\frac{2 \pi}{g^{2}} \sum_{i, n}\left|n+\frac{1}{2}\right|\left|y_{i, n+\frac{1}{2}}\right|^{2}\right],
$$

where we have used $y_{i, n+\frac{1}{2}}=y_{i,-n-\frac{1}{2}}^{*}$, which is a consequence of the reality of $y_{i}$. Equation (18) may be interpreted as a wave function of an infinite-dimensional quantum mechanics or equivalently $[11,12]$ as the Schrödinger wave functional of the compactified quantum field theory. One may observe that, as expected from a product of harmonic oscillators, states are exponentially confined to the classical vacuum with higher KK modes $n$ more strongly confined. In general the distance that states may wander from the vacuum is of order $g$.

The lightest modes are $n=-1$ and $n=0$, which are related by complex conjugation. Although this free truncation experiences corrections (to the exponential) of order unity far from the vacuum, one may crudely estimate the overlap of the two vacua by inserting $y \sim \pi / 2$ to conclude 
that indeed the overlap is of order $\exp \left(-c / g^{2}\right)$ for some $c$, as expected from a (fractional) instanton effect.

The generalization to a nonlinear sigma model with target space metric $g_{i j}$ is straightforward. In this case,

$$
\begin{aligned}
\Pi_{i} & =\frac{2}{g^{2}} g_{i j} \partial_{t} y_{j} \\
\mathcal{H} & =\sum_{i, j}\left(\frac{g^{2}}{4}: g^{i j} \Pi_{i} \Pi_{j}:+\frac{1}{g^{2}}: g_{i j} \partial_{x} y_{i} \partial_{x} y_{j}:\right),
\end{aligned}
$$

where $g^{i j}$ is the inverse metric. In the case of a $\mathbb{C P} \mathbb{P}^{1}$ model, we identify $y_{1}+i y_{2}$ with the affine coordinates for $\mathbb{C P}^{1}$. Now one classical vacuum is at the origin while the other lies at infinity. As the $\mathbb{C P}^{1}$ is a unit sphere, in affine coordinates the metric is given by four times the Fubini study metric:

$$
g_{i j}=\frac{4 \delta_{i j}}{\left(1+y_{1}^{2}+y_{2}^{2}\right)^{2}} .
$$

Let us now truncate our theory down to the four lowest KK modes, corresponding to $|n+1 / 2|=1 / 2$. Note that this truncation explicitly violates the $y_{1}+i y_{2} \rightarrow 1 /\left(y_{1}+\right.$ $\left.i y_{2}\right)$ symmetry which exchanges the vacua. Now our two quantum fields reduce to four-dimensional quantum mechanics via the decomposition

$y_{i}=\sqrt{\frac{L}{2 \pi}}\left[\left(b_{i,-\frac{1}{2}}+b_{i, \frac{1}{2}}^{\dagger}\right) e^{-i \frac{\pi}{L} x}+\left(b_{i, \frac{1}{2}}+b_{i,-\frac{1}{2}}^{\dagger}\right) e^{i \frac{\pi}{L} x}\right]$.

This four-dimensional theory is invariant under rotations of $\phi$ or equivalently $y_{1}+i y_{2}$. The low lying states will be rotation invariant and these are already sufficient to study the instantons. Therefore, we will fix the rotational freedom by setting $b_{1,1 / 2}=-b_{1,-1 / 2}$ so that $y_{1}$ is imaginary and equal to

$$
y_{1}=-2 i \sqrt{\frac{L}{2 \pi}}\left(b_{1, \frac{1}{2}}-b_{1, \frac{1}{2}}^{\dagger}\right) \sin \left(\frac{\pi}{L} x\right) .
$$

Physically, this means that the state reaches its maximal extent in $y_{1}$ at $|x|=L / 2$. By combining a rotation with a shift in $x$ we can also impose the condition $b_{2,1 / 2}=b_{2,-1 / 2}$ so that $y_{2,1 / 2}$ is real. This corresponds to an orbit in which $y_{1}$ and $y_{2}$ are the principle axes, with $y_{2}$ extremized at $x=0$ and vanishing at the boundaries. We are left with a twodimensional quantum mechanics in which the field $y_{2}$ has been decomposed as

$$
y_{2}=2 \sqrt{\frac{L}{2 \pi}}\left(b_{2, \frac{1}{2}}+b_{2, \frac{1}{2}}^{\dagger}\right) \cos \left(\frac{\pi}{L} x\right) .
$$

Now that the mode numbers are all equal to $1 / 2$, they will be omitted. The conjugate momenta may be decomposed:

$$
\begin{aligned}
\Pi_{i}= & \frac{2}{g^{2}} \sqrt{\frac{2 \pi}{L}} \\
& \times\left[g_{i 1}\left(b_{1}+b_{1}^{\dagger}\right) \sin \left(\frac{\pi}{L} x\right)-i g_{i 2}\left(b_{2}-b_{2}^{\dagger}\right) \cos \left(\frac{\pi}{L} x\right)\right] .
\end{aligned}
$$

Putting everything together, we obtain the Hamiltonian

$$
\begin{aligned}
& \mathcal{H}=\frac{8 \pi}{g^{2} L} \frac{\left[\left(b_{1}+b_{1}^{\dagger}\right)^{2}+\left(b_{2}+b_{2}^{\dagger}\right)^{2}\right] \sin ^{2}\left(\frac{\pi}{L} x\right)-\left[\left(b_{1}-b_{1}^{\dagger}\right)^{2}+\left(b_{2}-b_{2}^{\dagger}\right)^{2}\right] \cos ^{2}\left(\frac{\pi}{L} x\right)}{\left(1+4 \frac{L}{2 \pi}\left[-\left(b_{1}-b_{1}^{\dagger}\right)^{2} \sin ^{2}\left(\frac{\pi}{L} x\right)+\left(b_{2}+b_{2}^{\dagger}\right)^{2} \cos ^{2}\left(\frac{\pi}{L} x\right)\right]\right)^{2}}, \\
& H=\int_{x=-L / 2}^{L / 2} d x \mathcal{H}=\frac{4 \pi}{L} \frac{1}{\sqrt{\left(1+b_{2+}^{2}\right)\left(1+b_{1-}^{2}\right)}}\left[\frac{b_{1+}^{2}+b_{2+}^{2}}{1+b_{1-}^{2}}+\frac{b_{1-}^{2}+b_{2-}^{2}}{1+b_{2+}^{2}}\right],
\end{aligned}
$$

where we have defined

$b_{i+}=\frac{\sqrt{L}}{g}\left(b_{i}+b_{i}^{\dagger}\right), \quad b_{i-}=-i \frac{\sqrt{L}}{g}\left(b_{i}-b_{i}^{\dagger}\right)$.

Note that (25) has a simple interpretation as a Hamiltonian for two-dimensional quantum mechanics with coordinates $b_{1-}$ and $b_{2+}$ and momenta $-b_{1+}$ and $b_{2-}$. The isolated vacua are at $b_{1-}=b_{2+}=0$ and $b_{1-}=b_{2+}=\infty$. Using this truncated Hamiltonian, one may calculate the instanton contributions to the wave function and energies.
Unfortunately $\left[b_{i+}, b_{j-}\right]=i \delta_{i j}$ only near the vacuum at the origin $y=0$ and so in general these positions and momenta are not quite canonically conjugate. This is a result of the metric in the expression for $\Pi_{i}$ in Eq. (20), which differs from the identity matrix away from the origin.

In general the dynamics of this theory is quite complicated. The mode expansion truncation does not commute with the QFT Hamiltonian, although the difference is subleading in $g$, and so the dynamics of the truncated QM and the original QFT are generally inequivalent. One exception is the trajectories $b_{1-}=b_{2+}$, representing maps where the latitude is independent of $x$. Such trajectories 
interpolate between the vacua at infinity and zero. The half-charged instanton is of this form in the Euclidean theory.

Beyond the leading order interactions, the Hamiltonian (25) differs from that found in Refs. [5,6,8,9]. Note that Eq. (3.17) of Ref. [8] is not consistent with the condition that the field be restricted to the $\mathbb{C P}^{1}$, since the field $\tilde{n}$ in that equation is not in general a unit vector. This can be corrected by adding a constraint by hand to the Lagrangian [13], or by introducing a Lagrange multiplier [14] or Dirac constraints [15]. More importantly, as is explained under Eq. (4.20) of [8], in the reduction to quantum mechanics it is assumed that the latitude $\theta$ is constant. This implies that fixed time slices are circles of parallel in $\mathbb{C P}^{1}$, not geodesics, and so in general not the lowest energy curves with given boundaries. In other words, they only consider configurations with $b_{1-}=b_{2+}$, yielding a one-dimensional slice of the quantum mechanical system which includes the half-instantons of interest but not the lowest energy perturbative excitations.

\section{ACKNOWLEDGMENTS}

J. E. is supported by the CAS Key Research Program of Frontier Sciences Grant No. QYZDY-SSW-SLH006 and the NSFC MianShang Grants No. 11875296 and No. 11675223. J.E. also thanks the Recruitment Program of High-End Foreign Experts for support.
[1] V. A. Fateev, I. V. Frolov, and A. S. Shvarts, Nucl. Phys. B154, 1 (1979).

[2] J. Balog, S. Naik, F. Niedermayer, and P. Weisz, Phys. Rev. Lett. 69, 873 (1992).

[3] M. Hasenbusch and S. Meyer, Phys. Rev. Lett. 68, 435 (1992).

[4] K. Uhlenbeck, J. Diff. Geom. 30, 1 (1989).

[5] A. Cherman, D. Dorigoni, G. V. Dunne, and M. Ũnsal, Phys. Rev. Lett. 112, 021601 (2014).

[6] A. Cherman, D. Dorigoni, and M. Unsal, J. High Energy Phys. 10 (2015) 056.

[7] M. Nitta, J. High Energy Phys. 08 (2015) 063.
[8] G. V. Dunne and M. Unsal, J. High Energy Phys. 11 (2012) 170.

[9] G. V. Dunne and M. Unnsal, Phys. Rev. D 87, 025015 (2013).

[10] S. R. Coleman, Commun. Math. Phys. 31, 259 (1973).

[11] E. C. G. Stueckelberg, Helv. Phys. Acta 11, 225 (1938).

[12] K. O. Friedrichs, Commun. Pure Appl. Math. 4, 161 (1951).

[13] W. A. Bardeen, B. W. Lee, and R. E. Shrock, Phys. Rev. D 14, 985 (1976).

[14] A. D'Adda, M. Luscher, and P. Di Vecchia, Nucl. Phys. B146, 63 (1978).

[15] M. F. Lapa and T. L. Hughes, Phys. Rev. D 96, 045010 (2017). 